\title{
Ubicación y Operación Eficiente de Almacenadores de Energía en Micro-redes en Presencia de Generación Distribuida
}

\section{Optimal Location and Operation of Energy Storage Devices in Microgrids in Presence of Distributed Generation}

\author{
O. D. Montoya ${ }^{1}$, A. Grajales ${ }^{2}$, L. F. Grisales ${ }^{3}$ and C. A. Castro ${ }^{4}$
}

Recibido: 5 de enero 2017

Aceptado: 8 de junio 2017

\section{Resumen}

En este trabajo se propone una metodología eficiente para la localización y operación óptima de almacenadores de energía (AE) en micro-redes (MR) a través de un modelo de programación lineal entera mixta (MPLEM). El modelo matemático desarrollado corresponde a un modelo de despacho económico para miro-redes (DEMR) con presencia de generación distribuida (GD) a partir de recursos energéticos renovables. Como función objetivo se considera la minimización de los costos de compra de energía en la bolsa por parte del agente operador de red, teniendo en cuenta dos escenarios de operación; el primero considera un precio de energía fijo; y el segundo, precios variables en el nodo que conecta la MR al sistema de potencia para un horizonte operativo de 24 horas. Como restricciones se consideran los flujos de potencia por la línea a través de un modelo de flujo en redes, las capacidades de almacenamiento de los AE y las capacidades horarias de generación de potencia en los GD.

\footnotetext{
$1 \quad$ Universidad Tecnológica de Pereira, Pereira, Colombia

2 XM Compañía de Expertos en Mercados S.A.E.S.P, Medellín, Colombia

3 Instituto Tecnológico Metropolitano, Medellín, Colombia

$4 \quad$ Universidad Estatal de Campinas, Campinas, SP, Brasil

Correos: o.d.montoyagiraldo@ieee.org, luisgrisales@itm.edu.co, agrajales@xm.com.co and ccastro@ieee.org
} 
Para resolver el MPLEM propuesto se emplea el paquete de optimización comercial GAMS, empleando el solver CPLEX. La metodología propuesta permite localizar, dimensionar y operar los AE, considerando como horizonte de operación, un día típico laboral en Colombia, adicionalmente se tienen en cuenta diferentes consignas operativas en la operación de los $A E$ según los estándares existentes. Con el fin de conocer las variables de estado de la $M R$, es empleado un flujo de potencia del tipo barrido iterativo para evaluar las respuestas obtenidas por el MPLEM. Esta evaluación permite hallar los costos operativos reales de la MR. Para validar el modelo matemático propuesto es empleado un sistema de prueba de la literatura especializada de 7 nodos con presencia de dos generadores distribuidos, siendo uno de tipo fotovoltaico y el otro tipo eólico. Los resultados obtenidos muestran la eficiencia de la metodología propuesta, así como su facilidad de implementación y la capacidad de adaptación para sistemas de mayor tamaño.

Palabras clave: Almacenadores de energía, despacho económico, flujo de potencia, generación distribuida, micro-redes, programación lineal entera.

\section{Abstract}

In this paper, an efficient methodology for the location and optimal operation of power storage in micro-grids through a mixed integer linear programming model (MILPM) is proposed. The developed mathematical model corresponds to an economic dispatch model for micro-grid with presence of distributed generation from renewable energy resources. Minimization costs of energy purchase in the energy exchange by the independent system operator, taking into account two operating scenarios, is considered as objective function: First scenario considers a fixed energy price and the second, variable prices at the node that connects the MG to the power system for a 24-hour operating horizon. The power flow by the branches, the PS capacities and the hourly capacities of power generation in the DG are considered as constraints. The CPLEX solver of optimization package GAMS is used to solve the proposed MILPM. The proposed methodology allows to locate, sizing and operate the PS, considering a typical labor day in Colombia as a horizon of operation, besides taking into account different operational schemes of the PS according to the existing standards. To know the state variables of the MG, a backward/forward sweep method is used to evaluate the responses obtained by the LIMPM. This evaluation allows to find the actual operational costs of MG. To validate the proposed mathematical model, a 7-nodes test feeder with two distributed generators is used, being one of photovoltaic type and the other wind type. The results obtained show the efficiency of the proposed methodology, as well as its ease of implementation and the adaptability for larger systems

Keywords: Energy storage, economic dispatch, power flow, distributed generation, microgrids, linear integer programming 


\section{Acrónimos}

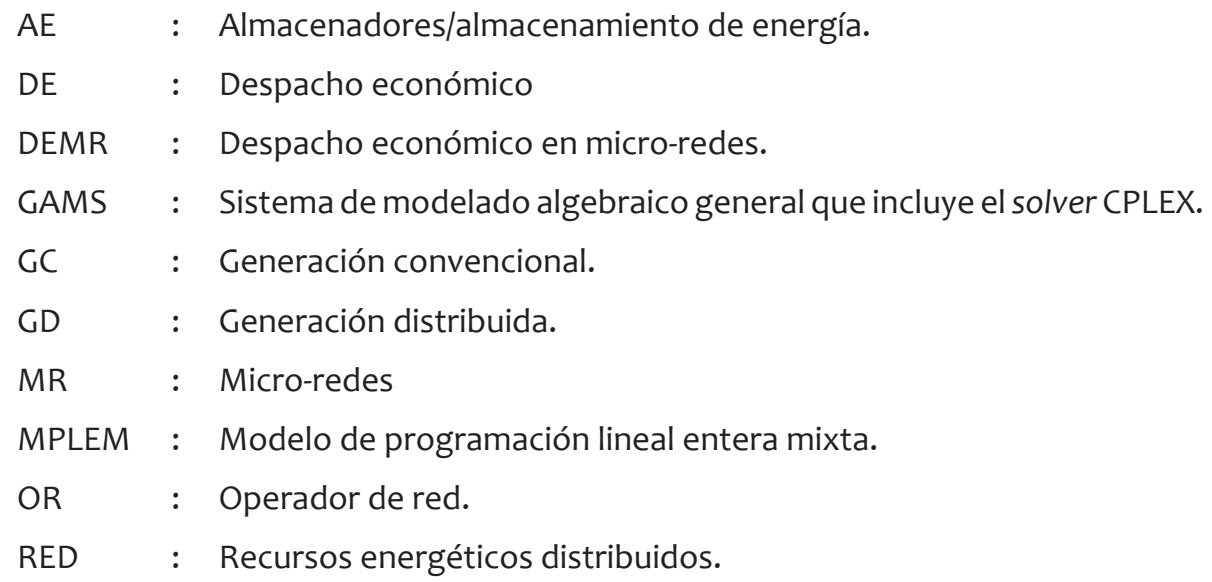

\section{Nomenclatura}

Conjuntos:

$\Omega_{B} \quad$ : Conjunto que contiene todos los tipos de AE disponibles.

$\Omega_{G} \quad$ : Subconjunto que contiene todos los nodos que tienen una fuente

$\Omega_{L} \quad$ : Conjunto que contiene todos los tramos de red.

$\Omega_{N} \quad$ : Conjunto que contiene todos los nodos de la red.

$\Omega_{T} \quad$ : Conjunto que contiene todos los intervalos de tiempo del período de estudio.

Índices:

$b \quad: \quad$ Índice asociado a los AE.

$i \quad$ : I Índice asociado a los nodos de la red.

ij : : Índice asociado a los tramos de red.

$i \quad$ : Índice asociado a los nodos de la red.

$t \quad$ : Índice asociado a los períodos de estudio.

Parámetros:

$C C(b) \quad$ : Costo de compra de un AE tipo $b[\$]$.

$C I_{B}^{\max } \quad$ : Límite máximo de inversión en $\mathrm{AE}$ [\$].

$C_{g c}(t, i) \quad$ : Costo de compra de energía en bolsa para el generador convencio- 
$E B_{\max }(b): \quad$ Límite máximo de almacenamiento de energía de un $\mathrm{AE}$ tipo $\mathrm{b}$

$E B_{\min }(b)$ : Límite mínimo de almacenamiento de energía de un AE tipo $b$

$P_{d}(t, i) \quad$ : Potencia activa demanda en el nodo i en el período $t$ [kW].

$F_{\max }(i j) \quad$ : Límite máximo de flujo de potencia activa entre los nodos i y j [kW].

$P_{g c}^{\max }(i) \quad$ : Límite máximo de generación de potencia activa de un generador

$P_{g c}^{\min }(i) \quad: \quad \begin{aligned} & \text { Límite mínimo de generación de potencia activa de un generador } \\ & \text { convencional conectado al nodo } i[\mathrm{~kW}] \text {. }\end{aligned}$

$P_{g d}^{\max }(i) \quad$ : Límite máximo de generación de potencia activa de un generador

$P_{g d}^{\min }(i) \quad$ : Límite mínimo de generación de potencia activa de un generador

: distribuido conectado al nodo $i[\mathrm{~kW}]$.

$\operatorname{SoC}_{F}(b, i)$ : Estado final de carga de un almacenador de energía tipo $b$ conec-

TC $(b) \quad$ : Tiempo de carga del AE tipo $b[\mathrm{~h}]$.

$T D(b) \quad$ : Tiempo de descarga del AE tipo $b[\mathrm{~h}]$.

$\Delta_{T} \quad: \quad$ Longitud de los períodos de tiempo considerados [h].

$\begin{array}{ll}\varphi(b) \quad & \text { Coeficiente de eficiencia asociado la carga/descarga de un AE tipo } \\ & b[\%] .\end{array}$

Variables continuas:

$P_{g c}(t, i) \quad$ : Potencia activa generada por un generador convencional conecta-

$P_{g d}(t, i) \quad: \quad \begin{aligned} & \text { Potencia activa generada por un generador distribuido conectado } \\ & \text { al nodo } i \text { en el período } t[\mathrm{~kW}] .\end{aligned}$

$P(t, b, i) \quad$ : $\quad \begin{aligned} & \text { Potencia activa generada/consumida por un } \mathrm{AE} \text { tipo } b \text { conectado al } \\ & \text { nodo } i \text { en el periodo } t[\mathrm{~kW}] \text {. }\end{aligned}$

$\operatorname{SoC}(t, b, i): \quad$ Estado de carga de un AE tipo $b$ conectado al nodo i en el período $t[\%]$.

Variables binarias:

$x(b, i) \quad$ : Variable de decisión que toma el valor de uno (1) si un AE tipo b es conectado al nodo i. En caso contrario toma el valor de cero (0). 


\section{Introducción}

El desarrollo tecnológico ha sido el pilar fundamental de la evolución de la sociedad en el siglo XXI, en este sentido los sistemas eléctricos se convierten en la base de todo el sistema económico y político que sustenta el crecimiento y desarrollo de las sociedades modernas en la llamada era de las tecnologías ${ }^{[1]}$. Sin embargo, existe a nivel mundial una preocupación creciente por el impacto que está dejando la globalización de la economía y la expansión de los mercados en el medio ambiente en todo el orbe ${ }^{[2]}$. Esta preocupación se refleja en el tratado firmado en París en diciembre de 2015, cuando líderes políticos de más de 190 países, firmaron un acuerdo guía para reducir los efectos del calentamiento global producido por la emisión de gases de efecto invernadero a la atmósfera ${ }^{[3-4]}$

En este sentido, y aunque el principal contaminador del medio ambiente corresponde al sistema de transporte convencional, el sistema eléctrico, mediante su parque térmico, es el segundo mayor contribuyente al calentamiento global [5-6]. Razón por la cual, uno de los objetivos propuestos es llevar las matrices energéticas de los países dependientes de combustibles fósiles (carbón, gas natural y petróleo) hacia un sistema compatible con el medio ambiente, como lo son las energías renovables, destacándose principalmente la generación solar térmica, solar fotovoltaica y eólica ${ }^{[2]}$.

Dada la creciente necesidad de desplazar paulatinamente los sistemas de generación convencional (predominantemente sistemas térmicos), por sistemas energéticos sostenibles y compatibles con el medio ambiente; en los últimos años se han venido desarrollando redes eléctricas menos dependientes de la generación térmica clásica ${ }^{[6]}$, las cuales incluyen GD, sistemas de AE y gestión eficiente de la demanda [8-4]. Por lo anterior, lo sistemas eléctricos actuales han migrado hacia un nuevo paradigma de operación conocido como redes inteligentes ${ }^{[15]}$.

Las redes inteligentes se conciben como sistemas que tienen la capacidad de auto-controlarse, tal que se maximiza la calidad y confiabilidad del servicio para los usuarios finales, al tiempo que se reducen las pérdidas de energía [15]. Dentro de este nuevo paradigma emergen las micro-redes (MR), como sistemas de pequeña escala auto-controlables que permiten la integración de las redes eléctricas convencionales con las fuentes alternativas de gene- 
ración de energía y los sistemas de $A E$, tal que se logra una gestión eficiente de la demanda de manera localizada ${ }^{[16-17]}$. En otras palabras, una red eléctrica inteligente está compuesta por un conjunto de MR conectadas de tal manera que pueden operar de forma autónoma o coordinada ${ }^{[18-19]}$.

La evolución de la electrónica de estado sólido y la reducción de costos en su proceso de producción, ha hecho posible la construcción de MR en diferentes partes del mundo ${ }^{[20-22]}$, mostrando así su potencial beneficio para la reducción del cambio climático y la gestión eficiente de la demanda de manera localizada [15], sin la necesidad de construir nuevos sistemas de transmisión de alto costo y grandes efectos sobre la vida silvestre o la inundación de grandes terrenos, para la construcción de nuevas centrales hidráulicas que, aunque no son causantes de emisiones de gases de efecto invernadero [7], generan desplazamiento forzado de comunidades, reducción de terrenos cultivables y desaparición de especies únicas de flora y fauna.

Por lo anterior, es posible encontrar en la literatura especializada, gran cantidad de trabajos que estudian el diseño, la operación y el control de MG, tal que se pueda maximizar la utilización de los recursos energéticos distribuidos, al tiempo que se mejoran los indicadores asociados a la calidad y confiabilidad en la prestación del servicio ${ }^{[18-19]}$. En este sentido, se encuentran propuestas relacionadas con la planeación de sistemas eléctricos de potencia en el mediano y largo plazo, por lo cual se proponen metodologías de ubicación de generadores distribuidos y sistemas de almacenamiento de energía en forma simultánea, cuyas funciones objetivo clásicas son la minimización de los costos de operación, o los costos de compra de energía [9-14]. Estos modelos son resueltos con diversas técnicas de optimización, entre las que sobresalen las técnicas metaheurísticas y los modelos de programación lineal y no lineal. También se encuentran trabajos relacionados con la operación del sistema, de entre los que se destacan estrategias como el DEMR y el flujo de potencia óptimo ${ }^{[18-19]}$.

Por último, se encuentran propuestas de control para MR que involucran el desarrollo de metodologías de control en los niveles primario ${ }^{[23-24]}$, secundario ${ }^{[25]}$ y terciario ${ }^{[26]}$. Estas estrategias de operación y dependen básicamente de las condiciones operativas de la MR y la red de potencia a la que se interconecta. Para lograr estas estrategias de control, son empleadas estrategias de control clásicas como: controladores proporcionales-integrales-de- 
rivativos (PID) y realimentación de variables de estado, con estrategias inteligentes como el control predictivo y los modelos de control no lineal [15], [27-28].

A diferencia de los trabajos anteriores, en este artículo se propone una metodología eficiente para la integración óptima de sistemas de AE en redes de distribución con alta penetración de generación distribuida. El problema es modelado como un despacho económico de MR, en el cual se pretende minimizar los costos de compra de energía en el mercado mayorista. Estos costos están relacionados de manera directa con los costos operativos de las centrales de generación térmica convencional. Para lo anterior se propone un modelo de programación lineal que garantiza una solución única en tiempos de cómputo susceptibles de aplicación en la toma de decisiones operativas en tiempo real. El modelo propuesto puede ser enmarcado dentro de las herramientas de control terciario, ya que se propone una adaptación del problema clásico de despacho económico en sistemas de potencia.

\section{Despacho económico en micro-redes}

Para entender la importancia del DE en la operación eficiente de MR, considérese una red eléctrica como la presentada en la Fig. 1, donde se puede observar la interconexión de recursos energéticos convencionales y recursos energéticos distribuidos ${ }^{[29]}$.

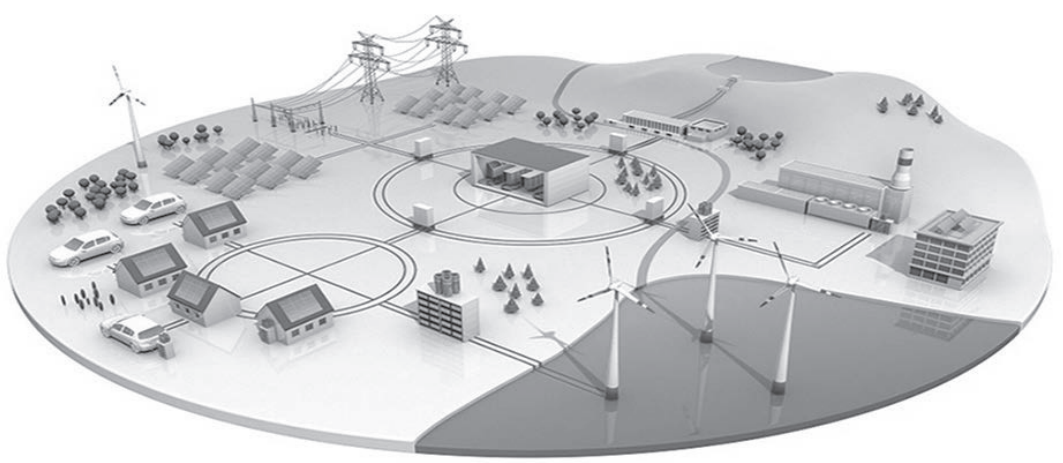

FIGURA 1. ESQUEMA TÍPICO DE UNA MR ${ }^{[29]}$

Para realizar el DEMR como la presentada en la Fig. 1, es necesario conocer los perfiles de demanda y generación para cada intervalo del período de 
estudio; así pues, no es posible pensar en intervalos de tiempo en el rango de días o semanas, dado que los patrones climáticos son de característica estocástica, y por tanto, su predicción a largo plazo es prácticamente inviable [9-11]. Es entonces necesario contar con herramientas que permitan hacer proyecciones de operación en intervalos de tiempo de horas y minutos, por lo cual el modelo de DE que se propone, para efectos de ubicación y operación óptima de $\mathrm{AE}$, está discretizada en periodos de tiempo de 24 horas, los cuales corresponden a un día típico de consumo ${ }^{[2-15]}$.

En razón a lo anterior, el problema de DEMR, es básicamente una extrapolación del problema de DE aplicado a los sistemas hidro-térmicos convencionales ${ }^{[18-19]}$. En este sentido, el objetivo fundamental del trabajo consiste en minimizar el costo de compra de energía por parte del operador de red en la bolsa de energía, lo cual es equivalente a minimizar los costos de generación de las centrales térmicas, ya que los $\mathrm{AE}$ tienen la capacidad de almacenar energía en horas de bajo costo, con el fin de atender a la demanda en horas de alto costo de operación (períodos de demanda pico).

Por otro lado, es aprovechada la capacidad de generación de los recursos energéticos distribuidos para atender a la demanda cuando existen suficientes recursos energéticos primarios (alta radiación solar y altas velocidades del viento), lo cual le permite a los AE tomar energía desde las centrales hidráulicas de bajo costo y emular la capacidad que tienen estas centrales para almacenar energía mediante el embalse [30-31].

En la literatura especializada, es común encontrar modelos de DEMR en los cuales se tienen en cuenta los costos de compra de energía en bolsa ${ }^{[18-19]}$, costos de generación con centrales diésel o reducción de emisiones de gases de efecto invernadero, aprovechamiento eficiente de energías renovables y operación eficiente considerando sistemas de AE [9-15].

Los modelos de DE mencionados, normalmente consideran que la red ya está diseñada y no proponen la ubicación de ninguna tecnología en conjunto con un modelo de DE eficiente, lo cual impacta de manera directa el esquema de operación obtenido, debido a que no existe posibilidad de seleccionar los mejores puntos de localización de los recursos energéticos distribuidos para beneficio de la red [18-19], [30-31]. En este sentido, los puntos de ubicación de los recursos energéticos distribuidos son definidos en función del conocimiento experto de los operadores de red que, siendo de suma importancia para el 
funcionamiento adecuado del sistema eléctrico, pueden causar sobrecostos de inversión, ya que, al ser una solución heurística, no corresponde necesariamente a la mejor solución técnico-económica que puede ser encontrada.

Así pues, el modelo que se propone para la localización óptima de AE integrada con un modelo de DEMR tiene la capacidad de operar como un modelo estático o dinámico (ver sección 3), es decir, sirve para planear la ubicación de sistemas de AE y/o para definir el mejor esquema de operación en función de la disponibilidad de recursos energéticos renovales y los costos de compra de energía en bolsa, lo cual le da la capacidad al operador de red de maximizar la relación beneficio/costo de la red, al tiempo que recibe incentivos económicos por parte del agente regulador del mercado, gracias a la sustitución de recursos energéticos de origen fósil por recursos energéticos alternativos.

\section{Formulación matemática}

Para formular el problema de DEMR con presencia de generación distribuida y almacenamiento de energía se emplea un MPLEM mono-objetivo. Este modelo minimiza los costos de compra de energía en bolsa en los que incurre el operador de la red, por medio del aprovechamiento eficiente de los recursos energéticos renovables, con la inclusión de un sistema de almacenamiento de energía que permita almacenar energía en horas de alta generación y baja demanda, para suministrarla a los usuarios en horas de baja generación renovable y alta demanda ${ }^{[18-19]}$. Como restricciones del problema son consideradas las capacidades máximas de los generadores distribuidos, límites de almacenamiento de energía, costos de inversión y una representación de la red, mediante un modelo de flujo DC. El modelo matemático desarrollado se presenta de (1) a (9).

Función objetivo:

$$
\begin{aligned}
& \min z=\left\{\sum_{t \in \Omega_{T}} \sum_{i \in \Omega_{G}} C_{g c}(t, i) \cdot P_{g c}(t, i) \cdot \Delta_{T}\right\} \\
& P_{g c}(t, i)+P_{g d}(t, i)+\sum_{b \in \Omega_{B}} P(t, b, i) \\
&=\sum_{i j \in \Omega_{L}, j \neq i} f(t, i j)+P_{d}(t, i)
\end{aligned}
$$




$$
\begin{aligned}
& \left\{\forall t \in \Omega_{T}-\forall i \in \Omega_{N}-\forall i j \in \Omega_{L}\right\} \\
& \operatorname{SoC}(t, b, i)+\varphi(b) \cdot P_{b}(t, b, i) \\
& =x(b, i) \cdot \operatorname{SoC}_{b}(t-1, b, i) \\
& \left\{\forall t \in \Omega_{T}-\forall i \in \Omega_{N}-\forall b \in \Omega_{B}\right\} \\
& \operatorname{SoC}(t, b, i)=x(b, i) \cdot \operatorname{SoC}_{I}(b, i) \\
& \left\{[t=1]-\forall i \in \Omega_{N}-\forall b \in \Omega_{B}\right\} \\
& \operatorname{SoC}(t, b, i)=x(b, i) \cdot \operatorname{SoC}_{F}(b, i) \\
& \left\{[t=24]-\forall i \in \Omega_{N}-\forall b \in \Omega_{B}\right\} \\
& P_{g c}^{\min }(i) \leq P_{g c}(t, i) \leq P_{g c}^{\max }(i) \\
& P_{g d}^{\min }(i) \leq P_{g d}(t, i) \leq P_{g d}^{\max }(i) \\
& -F_{\max }(i j) \leq f(t, i j) \leq F_{\max }(i j) \\
& \left\{\forall b \in \Omega_{B}-\forall i \in \Omega_{N}-\forall i j \in \Omega_{L}\right\} \\
& S o C_{\text {min }}(b) \leq \operatorname{SoC}(t, b, i) \leq S o C_{\text {max }}(b) \\
& P(t, b, i) \geq x(b, i) \cdot E B_{\text {min }}(b) / \Delta_{T} \\
& P(t, b, i) \leq x(b, i) \cdot E B_{\max }(b) / \Delta_{T} \\
& \left\{\forall b \in \Omega_{B}-\forall i \in \Omega_{N}-\forall b \in \Omega_{B}\right\} \\
& \sum_{b \in \Omega_{B}} x(b, i) \leq 1 \quad\left\{\forall i \in \Omega_{N}\right\} \\
& x(b, i) \in(0,1) \quad\left\{\forall i \in \Omega_{N}-\forall b \in \Omega_{B}\right\} \\
& \sum_{i \in \Omega_{N}} \sum_{b \in \Omega_{B}} C C(b) \cdot x(b, i) \leq C I_{B}^{\max }
\end{aligned}
$$

El modelo matemático presentado en este trabajo ha sido desarrollado con base a los fundamentos de la investigación de Montoya et al ${ }^{[18]}$. Así pues, la expresión (1) define la función objetivo del problema y corresponde a la minimización de los costos de compra de energía en bolsa por parte del operador, y sólo corresponde al valor ofertado por las centrales térmicas, lo cual sólo refiere al costo de la energía generada por las fuentes convencionales. Por otro lado, la expresión (2) modela el balance de potencia activa en todas las barras del sistema; mientras que en (3) se 
determina el nivel de carga de cada $\mathrm{AE}$, como función de la potencia que entrega/consume de la red y la eficiencia de la batería. En (4) se muestra la forma de asignar un valor de carga inicial al $A E$, y de ser necesario, definir una meta final de almacenamiento para el período de operación previsto. Por su parte, la expresión (5) muestra los límites de capacidad de generación de potencia activa de la generación convencional y la generación distribuida, así como los límites de capacidad de las líneas que interconectan el sistema de potencia. La restricción (6) define que, cada AE sólo puede almacenar energía entre un valor mínimo de capacidad y su valor nominal, es decir, que existen condiciones operativas que impiden superar las capacidades de diseño del AE, así como condiciones mínimas de carga que protegen el $A E$ de la posibilidad de ocurrencia de fenómenos electroquímicos adversos ${ }^{[18]}$; adicionalmente, se muestra que la potencia activa que un $\mathrm{AE}$ puede entregar, está limitada por la capacidad máxima de diseño y es diferente de cero, sólo si se decide su instalación en el sistema. En la expresión (7) se garantiza que por cada nodo sólo pueda existir un AE. En (8) se presenta la naturaleza binaria de las variables de decisión, respectivamente. Por último, la ecuación (9) define el máximo nivel de inversión disponible para compra de AE.

Es de tener en cuenta que el modelo matemático presentado corresponde al MPLEM que se propone resolver mediante el paquete de optimización comercial GAMS y el solver CPLEX. Sin embargo, para evaluar las condiciones operativas reales del sistema de distribución, es necesario plantear un flujo de potencia del tipo barrido iterativo como el presentado en [32]. Es necesario aclarar que la solución obtenida en GAMS al resolver el $\mathrm{DE}$, corresponde a la etapa de ubicación óptima de $\mathrm{AE}$, así como su esquema de operación. Con esta solución, es inicializado el problema de flujo de potencia, el cual permite determinar las características operativas del sistema de distribución para cada intervalo de tiempo contenido en el periodo de estudio.

\section{Sistema de prueba}

Para verificar el modelo matemático propuesto, se considera un sistema de prueba de 8 nodos que se presenta en la Fig. 2, que ha sido tomado de ${ }^{[18]}$. 


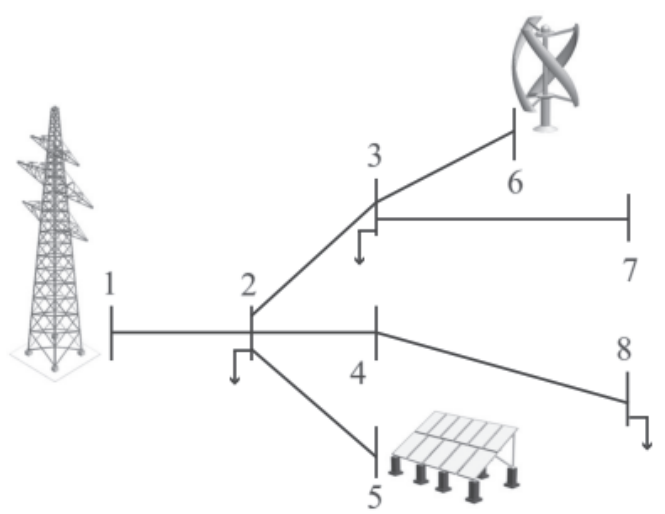

FIGURA 2. SISTEMA DE DISTRIBUCIÓN RADIAL ${ }^{[18]}$

Este sistema tiene un comportamiento típico de un sistema eléctrico colombiano, ya que la demanda corresponde a una curva de demanda típica para un día ordinario y los niveles de capacidad de generación eólica y fotovoltaica corresponden a una zona con niveles de radiación y viento similares a los que se presentan en las zonas ecuatoriales. A este sistema se le asocia un nivel de tensión de $2 \mathrm{kV}$ y se asume que todos los conductores son uniformes y con un valor de impedancia igual a $0.8763+j 0.4133 \Omega$.

Para este sistema, los datos de generación y demanda se presentan en la Tabla 1, teniendo en cuenta que para el nodo 2 se asigna el 30\% de la demanda, para el nodo 3 el $25 \%$ y para el nodo 8 el $45 \%$ restante ${ }^{[18]}$.

TABLA 1. DATOS DE GENERACIÓN Y DEMANDA

\begin{tabular}{|c|c|c|c|c|c|}
\hline Hora & $C_{g c}(t, i)[\$ / \mathrm{kWh}]$ & $P_{P V}^{\max }(i)[\mathrm{kW}]$ & $P_{W T}^{\max }(i)[\mathrm{kW}]$ & $P_{d}(i)[\mathrm{kW}]$ & $Q_{d}(i)[\mathrm{kVA}]$ \\
\hline 1 & 0,77 & 10 & 80 & 120 & 59 \\
\hline 2 & 0,71 & 10 & 80 & 108 & 44 \\
\hline 3 & 0,69 & 20 & 70 & 102 & 37 \\
\hline 4 & 0,70 & 20 & 50 & 100 & 32 \\
\hline 5 & 0,72 & 20 & 100 & 101 & 30 \\
\hline 6 & 0,80 & 10 & 110 & 107 & 30 \\
\hline 7 & 0,84 & 20 & 100 & 112 & 19 \\
\hline
\end{tabular}




\begin{tabular}{|c|c|c|c|c|c|}
\hline Hora & $C_{g c}(t, i)[\$ / k W h]$ & $P_{P V}^{\max }(i)[\mathrm{kW}]$ & $P_{W T}^{\max }(i)[\mathrm{kW}]$ & $P_{d}(i)[\mathrm{kW}]$ & $Q_{d}(i)[\mathrm{kVA}]$ \\
\hline 8 & 0,87 & 20 & 95 & 128 & 28 \\
\hline 9 & 0,91 & 40 & 80 & 154 & 46 \\
\hline 10 & 0,88 & 100 & 85 & 171 & 61 \\
\hline 11 & 0,91 & 180 & 110 & 188 & 75 \\
\hline 12 & 0,91 & 250 & 130 & 196 & 87 \\
\hline 13 & 0,91 & 150 & 180 & 200 & 92 \\
\hline 14 & 0,90 & 60 & 210 & 190 & 93 \\
\hline 15 & 0,89 & 30 & 200 & 195 & 90 \\
\hline 16 & 0,90 & 20 & 160 & 197 & 85 \\
\hline 17 & 0,95 & 20 & 150 & 197 & 83 \\
\hline 18 & 0,94 & 20 & 130 & 192 & 85 \\
\hline 19 & 0,91 & 20 & 120 & 189 & 108 \\
\hline 20 & 0,90 & 20 & 112 & 210 & 126 \\
\hline 21 & 0,85 & 20 & 100 & 205 & 127 \\
\hline 22 & 0,80 & 20 & 90 & 190 & 113 \\
\hline 23 & 0,71 & 20 & 85 & 166 & 89 \\
\hline 24 & 0,66 & 20 & 70 & 142 & 69 \\
\hline
\end{tabular}

Para este sistema de prueba existe la posibilidad de ubicar sistemas de $A E$ en todos los nodos, teniendo en cuenta que sólo se puede ubicar uno por cada nodo. Los datos de costo, capacidad y eficiencia de carga de los AE se presentan en la Tabla 2.

TABLA 2. CARACTERÍSTICAS DE LOS AE

\begin{tabular}{|c|c|c|c|c|c|c|}
\hline $\begin{array}{c}\text { Tipo } \\
\text { de AE }\end{array}$ & $C C(b)[\$]$ & $E B_{\max }(b)[\mathrm{kW}]$ & $E B_{\min }(b)[\mathrm{kW}]$ & $\varphi(b)[\%]$ & $T C(b)[\mathrm{h}]$ & $T D(b)[\mathrm{h}]$ \\
\hline 1 & 20000 & 62,5 & 50 & 0,4 & 4 & 5 \\
\hline 2 & 25000 & 50 & 40 & 0,5 & 4 & 5 \\
\hline 3 & 30000 & 40 & 40 & 0,5 & 5 & 5 \\
\hline
\end{tabular}


Es importante resaltar que los AE presentados en la Tabla 2, corresponden a baterías del tipo plomo-ácido como las reportadas en ${ }^{[19]}$.

\section{Aplicación y resultados}

Para evaluar el desempeño del modelo matemático propuesto se consideran dos escenarios de simulación sobre el sistema de prueba. El primer escenario se evalúa el caso base considerando la presencia de los generadores distribuidos, además de costos fijos y costos variables en el nodo de red. En el segundo escenario se considera que existe una restricción económica, en la cual la máxima inversión en AE, por parte del operador de red, es \$100.000 y se evalúa el problema considerando los costos fijos y los costos variables en el nodo de red.

Es de tener en cuenta que para cada uno de los escenarios propuestos se calcula la solución matemática del problema empleando el paquete de optimización comercial GAMS con el solver CPLEX, y posteriormente se evalúa el flujo de potencia del tipo barrido iterativo programado en el software MATLAB.

\subsection{Escenario 1}

En este escenario de prueba se evalúa el caso base del sistema de prueba. En este caso, es considerado que el costo asignado al nodo equivalente de red (ver nodo 1 de la Fig. 2) corresponde al costo de compra de energía presentado en la columna 2 de la Tabla 1. Para los costos fijos los autores asumen un valor constante equivalente al valor promedio de los costos variables. Adicionalmente, se realiza un DE para la red considerando que el nodo slack no puede vender energía al sistema de potencia. En la Tabla 3 se presentan los resultados del caso base para el MPLEM formulado en GAMS y su equivalente no lineal en MATLAB cuando es evaluada la solución obtenida por el proceso de optimización.

TABLA 3. Resultados del CASO BASE

\begin{tabular}{|c|c|c|c|}
\hline \multicolumn{2}{|c|}{ Función objetivo con costos fijos [\$] } & \multicolumn{2}{|c|}{ Función objetivo con costos variables [\$] } \\
\hline GAMS & MATLAB & GAMS & MATLAB \\
\hline 660,024 & 694,610 & 671,910 & 689,137 \\
\hline
\end{tabular}




\subsection{Escenario 2}

En este escenario se considera que existe una restricción económica en la red, tal que sólo es posible invertir en AE hasta un máximo de \$100.000, lo cual implica que la cantidad de AE que pueden ser ubicados está entre 3 y 5 .

TABLA 4. SOLUCIÓN ÓPTIMA PARA UN LÍMITE DE INVERSIÓN EN AE EQUIVALENTE A UNA INVERSIÓN DE \$100.000

\begin{tabular}{|c|c|c|c|c|c|c|}
\hline \multirow{2}{*}{$\begin{array}{c}\text { Tipos de } \\
\text { costos }\end{array}$} & \multirow{2}{*}{$\begin{array}{c}\text { Número } \\
\text { de } A E\end{array}$} & Tipo & \multirow{2}{*}{$\begin{array}{c}\text { Costo AE } \\
{[\$]}\end{array}$} & Ubicación & \multicolumn{2}{|c|}{ Función objetivo $[\$]$} \\
\cline { 7 - 8 } & & & Nodos) & GAMS & MATLAB \\
\hline Fijos & 3 & 1 & 90.000 & $1,2,3$ & 284,882 & 530,930 \\
\hline Variables & 3 & 1 & 90.000 & $1,2,3$ & 236,159 & 677,860 \\
\hline
\end{tabular}

\subsection{Análisis comparativo}

Al analizar los resultados obtenidos para el sistema de prueba considerando el caso base (ver Tabla 3), es decir, red sin presencia de AE, se observa que el MPLEM tiene en cuenta un despacho económico, que al ser evaluado en flujo de potencia convencional, presenta costos operativos muy similares. Estas diferencias entre MATLAB y GAMS se explican básicamente porque el MPLEM no considera el flujo de potencia reactiva por la red, es decir, desprecia las pérdidas de energía causadas por el flujo de corriente reactiva; mientras que en modelo de flujo de potencia evaluado en MATLAB, las pérdidas son asumidas por el nodo fuente, lo cual, naturalmente, incrementa sus costos de operación.

Por otro lado, al considerar la ubicación óptima de AE tomando en cuenta costos de inversión y con una limitante de inversión en términos de adquisición de sistemas de AE, se obtienen los resultados presentados en la Tabla 4. En este sentido, las soluciones propuestas para el MPLEM encuentran que con tres (3) AE es posible cumplir la restricción de inversión al tiempo que se minimizan los costos de operación del sistema. Sin embargo, al evaluar la solución propuesta el MPLEM en MATLAB, se encuentra que la solución, considerando costos fijos de compra de energía, mejora la respuesta presentada para el caso base en un valor de $\$ 163.680$. Lo cual implica que se requiere de un período de operación continua de aproximadamente 1.5 
años para recuperar la inversión. Adicionalmente, al analizar el caso de la ubicación de $\mathrm{AE}$ considerando costos variables, el resultado de ubicar tres (3) AE mejora ligeramente los costos operativos, en relación con el caso base. Esta mejora diaria corresponde a \$11.277; lo cual implica que bajo este escenario de prueba se requiere de 21.86 años para recuperar la inversión realizada. Lo anterior quiere decir que la solución obtenida, desde el punto de vista técnico, no es viable para un sistema con precio de energía variable, tomando en cuenta que los tiempos de planeamiento para sistemas de distribución están entre 10 y 20 años [33], lo cual es claramente superado por la respuesta alcanzada.

Es de notarse entonces que el MPLEM, para el caso de costos variables en el nodo slack, presenta una solución evidentemente atractiva para el operador de red; sin embargo, esta solución implica que los AE se cargan de la energía excedente de la GD y de la red cuando existe poca demanda y el costo de compra de energía en el nodo slack es lo suficientemente bajo. No obstante, al evaluar este escenario en el flujo de potencia de la red, se encuentra que esta compra de energía puede incrementar significativamente el valor de las pérdidas técnicas, por lo cual el beneficio obtenido por la atención de la demanda con la GD y el AE se cancela con el costo que asume la red para suplir lar pérdidas técnicas.

\section{Conclusiones y trabajos futuros}

Se desarrolló un modelo de programación lineal entera mixta que permite la ubicación óptima de almacenadores de energía en sistemas de distribución para reducir el costo de compra de energía en la bolsa, por medio de un modelo de despacho económico para micro-redes que considera la presencia de generación distribuida a base de energías renovables.

El modelo matemático propuesto, sirve como un indicador de sensibilidad para la selección óptima de sistemas de $A E$ en redes de distribución; sin embargo, es necesario considerar el impacto que tienen las simplificaciones realizadas, como es el hecho de no considerar la potencia reactiva dentro del MPLEM o suponer que las tensiones en todos los nodos del sistema son planos, es decir, que toman el valor de 1 en por unidad. 
La ubicación de sistemas de AE de energía a la luz de los resultados obtenidos, corresponde a una solución atractiva para el sistema de distribución, siempre que existan excedentes de generación en los generadores distribuidos; puesto que esta energía puede ser almacenada en horas de alta generación renovable y poca demanda, para luego ser suministrada a los usuarios del servicio en horas de alta demanda y baja generación distribuida.

No es aconsejable permitir que los sistemas de AE se carguen empleando como fuente de energía el nodo de la red en horas de bajo costo de energía, para luego ser entregada a la red en horas de alto consumo, debido a que el proceso de carga aumenta considerablemente el nivel de pérdidas técnicas, lo cual de manera inevitable aumenta los costos de operación del sistema, anulando el beneficio obtenido por la implementación de sistemas de $A E$.

Se recomienda incluir en la formulación del MPLEM, términos asociados a los efectos causados por la potencia reactiva, en términos de pérdidas técnicas, y considerar en el modelo matemático el impacto de la impedancia asociada a las ramas de distribución a través de una formulación que considere algunas características del flujo AC.

\section{Agradecimientos}

Al programa de Becas Doctorados Nacionales del Departamento Administrativo de Ciencia, Tecnología e Innovación de Colombia (COLCIENCIAS), según convocatoria 727 de 2015 y al trabajo conjunto de los investigadores del grupo investigación DINOP de la Universidad Tecnológica de Pereira y del grupo de investigación MATyER del Instituto Tecnológico Metropolitano

\section{Referencias}

[1] O. D. Montoya, A. Grajales, A. Garces and C. A. Castro, "Distribution Systems Operation Considering Energy Storage Devices and Distributed Generation," in IEEE Latin America Transactions, vol. 15, no. 5, pp. 890-900, May 2017. doi: 10.1109/ TLA.2017.7910203

[2] United Nations, "Adoption of the Paris Agreement," framework convention of climate change, pp. 1-32, Dec. 2015. 
Montoya et, al / Ubicación y Operación Eficiente de Almacenadores de Energía en Micro-redes...

[3] R. Mahabir and R. M. Shrestha, "Climate change and forest management: Adaptation of geospatial technologies," Agro-Geoinformatics (Agro-geoinformatics), 2015 Fourth International Conference on, Istanbul, 2015, pp. 209-214. Doi: 10.1109/ Agro-Geoinformatics.2015.7248108

[4] A. M. Hasna, "Climate Change, Technology, and Sustainability," in IEEE Technology and Society Magazine, vol. 29, no. 4, pp. 30-36, winter 2010.doi: 10.1109/ MTS.2010.939226

[5] V. Azad and R. Khoie, "Mitigating carbon dioxide emission with gradual implementation of distributed generation in Northern California," North American Power Symposium (NAPS), 2013, Manhattan, KS, 2013, pp. 1-6. Doi: 10.1109/ NAPS.2013.6666898

[6] A. M. Hernandez and H. L. Reyes, "Behavior of Fuels Supply Variable Costs in Electrical Energy Generation using Gas and Coal," in IEEE Latin America Transactions, vol. 13, no. 9, pp. 2915-2921, Sept. 2015. Doi: 10.1109/TLA.2015.7350039

[7] H. W. Whittington and S. W. Gundry, "Global climate change and hydroelectric resources," in Engineering Science and Education Journal, vol. 7, no. 1, pp. 29-34, Feb 1998. Doi: 10.1049/esej:19980107

[8] M. J. Hossain, T. K. Saha and N. Mithulananthan, "Impacts of wind and solar integrations on the dynamic operations of distribution systems," Universities Power Engineering Conference (AUPEC), $201121^{\text {st }}$ Australasian, Brisbane, QLD, 2011, pp. 1-6.

[9] A. De Souza et al., "Microgrids operation with micro dispersed generators and renewables," Integration of Renewables into the Distribution Grid, CIRED 2012 Workshop, Lisbon, 2012, pp. 1-4. Doi: 10.1049/cp.2012.0866

[10] C. Abbey and G. Joos, "Coordination of Distributed Storage with Wind Energy in a Rural Distribution System,” Industry Applications Conference, 2007.42 ${ }^{\text {nd }}$ IAS Annual Meeting. Conference Record of the 2007 IEEE, New Orleans, LA, 2007, pp. 10871092. Doi: 10.1109/07IAS.2007.169

[11] Z. Wang, C. Gu, F. Li, P. Bale and H. Sun, "Active Demand Response Using Shared Energy Storage for Household Energy Management," in IEEE Transactions on Smart Grid, vol. 4, no. 4, pp. 1888-1897, Dec. 2013. Doi: 10.1109/TSG.2013.2258046

[12] S. I. Kampezidou, E. Polymeneas and S. Meliopoulos, "The economic effect of storage in systems with high penetration of renewable sources," North American Power Symposium (NAPS), 2015, Charlotte, NC, 2015, pp. 1-6. Doi: 10.1109/ NAPS.2015.7335207 
[13] M. Martinez, M. G. Molina and P. E. Mercado, "Optimal Storage Technology Selection and Sizing for Providing Reserve to Power Systems with High Penetration of Wind Generation," in IEEE Latin America Transactions, vol. 13, no. 9, pp. 2983-2990, Sept. 2015. Doi: 10.1109/TLA.2015.7350049

[14] G. Carpinelli, G. Celli, S. Mocci, F. Mottola, F. Pilo and D. Proto, "Optimal Integration of Distributed Energy Storage Devices in Smart Grids," in IEEE Transactions on Smart Grid, vol. 4, no. 2, pp. 985-995, June 2013. Doi: 10.1109/TSG.2012.2231100

[15] S. Parhizi, H. Lotfi, A. Khodaei and S. Bahramirad, "State of the Art in Research on Microgrids: A Review," in IEEE Access, vol. 3, no. , pp. 890-925, 2015. Doi: 10.1109/ ACCESS.2015.2443119

[16] S. Park, J. Lee, S. Bae, G. Hwang and J. K. Choi, “Contribution-Based Energy-Trading Mechanism in Microgrids for Future Smart Grid: A Game Theoretic Approach," in IEEE Transactions on Industrial Electronics, vol. 63, no. 7, pp. 4255-4265, July 2016. Doi: 10.1109/TIE.2016.2532842

[17] N. Nikmehr and S. Najafi Ravadanegh, "Optimal Power Dispatch of Multi-Microgrids at Future Smart Distribution Grids," in IEEE Transactions on Smart Grid, vol. 6, no. 4, pp. 1648-1657, July 2015. Doi: 10.1109/TSG.2015.2396992

[18] O. D. Montoya, A. Garcés and C. A Castro, "Operación eficiente de micro-redes en presencia de generadores distribuidos y almacenadores de energía," in SBSE2016, VI Simpósio Brasileiro de Sistemas Elétricos, Natal, RN, Brasil, CA, 2016, pp. 1-6.

[19] A. C. Luna, N. L. Diaz, F. Andrade, M. Graells, J. M. Guerrero and J. C. Vasquez, "Economic power dispatch of distributed generators in a grid-connected microgrid," $20159^{\text {th }}$ International Conference on Power Electronics and ECCE Asia (ICPE-ECCE Asia), Seoul, 2015, pp. 1161-1168. Doi: 10.1109/ICPE.2015.7167927

[20] S. Bracco, F. Delfino, M. Rossi and M. Robba, "A multi-objective optimization tool for the daily management of sustainable smart microgrids: Case Study: the savona campus SPM and SEB facilities," 2016 International Symposium on Power Electronics, Electrical Drives, Automation and Motion (SPEEDAM), Anacapri, 2016, pp. 683688. Doi: 10.1109/SPEEDAM.2016.7526018

[21] M. F. Z. Souza, "On rural microgrids design - a case study in Brazil," Innovative Smart Grid Technologies Latin America (ISGT LATAM), 2015 IEEE PES, Montevideo, 2015, pp. 160-164. Doi: 10.1109/ISGT-LA.2015.7381146

[22] F. Guo and C. Wen, "Distributed control subject to constraints on control inputs: A case study on secondary control of droop-controlled inverter-based microgrids," 
Montoya et, al / Ubicación y Operación Eficiente de Almacenadores de Energía en Micro-redes...

$20149^{\text {th }}$ IEEE Conference on Industrial Electronics and Applications, Hangzhou, 2014, pp. 1119-1124. Doi: 10.1109/ICIEA.2014.6931333

[23] S. M. Ashabani and Y. A. R. I. Mohamed, "A Flexible Control Strategy for Grid-Connected and Islanded Microgrids With Enhanced Stability Using Nonlinear Microgrid Stabilizer," in IEEE Transactions on Smart Grid, vol. 3, no. 3, pp. 1291-1301, Sept. 2012. Doi: 10.1109/TSG.2012.2202131

[24] E. Unamuno and J. A. Barrena, "Equivalence of primary control strategies for AC and DC microgrids," 2016 IEEE $16^{\text {th }}$ International Conference on Environment and Electrical Engineering (EEEIC), Florence, Italy, 2016, pp. 1-5. Doi: 10.1109/ EEEIC.2016.7555729

[25] A. Toliyat and A. Kwasinski, "Energy storage sizing for effective primary and secondary control of low-inertia microgrids," 2015 IEEE $6^{\text {th }}$ International Symposium on Power Electronics for Distributed Generation Systems (PEDG), Aachen, 2015, pp. 1-7. Doi: 10.1109/PEDG.2015.7223077

[26] E. R. Sanseverino et al., "Energy Management Systems and tertiary regulation in hierarchical control architectures for islanded microgrids," Environment and Electrical Engineering (EEEIC), 2015 IEEE 15 $5^{\text {th }}$ International Conference on, Rome, 2015, pp. 144-149. Doi: 10.1109/EEEIC.2015.7165525

[27] F. Salem and M. I. Mosaad, "A comparison between MPC and optimal PID controIlers: Case studies,” Michael Faraday IET International Summit 2015, Kolkata, 2015, pp. 59-65. Doi: 10.1049/cp.2015.1607

[28] A. Bidram, A. Davoudi, F. L. Lewis and J. M. Guerrero, "Distributed Cooperative Secondary Control of Microgrids Using Feedback Linearization," in IEEE Transactions on Power Systems, vol. 28, no. 3, pp. 3462-3470, Aug. 2013. Doi: 10.1109/ TPWRS.2013.2247071

[29] 3M science applied to life, "Smart Grid Connected, Efficient and Sustainable Energy" available in: http://solutions.3m.com/wps/portal/3M/en_EU/SmartGrid/EU-SmartGrid/

[30] N. Yan, Z. X. Xing, W. Li and B. Zhang, "Economic Dispatch Application of Power System With Energy Storage Systems," in IEEE Transactions on Applied Superconductivity, vol. 26, no. 7, pp. 1-5, Oct. 2016. Doi: 10.1109/TASC.2016.2598963

[31] S. S. Haroon and T. N. Malik, "Environmental economic dispatch of hydrothermal energy system using stud differential evolution," 2016 IEEE $16^{\text {th }}$ International Conference on Environment and Electrical Engineering (EEEIC), Florence, Italy, 2016, pp. 1-5. Doi: 10.1109/EEEIC.2016.7555630 
[32] D. Shirmoharmnadi, H. Hong, A. Semlyen and G. Luo. "A compensation-based power flow method for weakly meshed distribution and transmission networks". IEEE Transactions on Power Systems. Vol. 3, pp. 753-762. May, 1988. ISSN: 08858950. DOI: 10.1109/59.192932.

[33] M. Lavorato, J. F. Franco, M. J. Rider and R. Romero, "Imposing Radiality Constraints in Distribution System Optimization Problems," in IEEE Transactions on Power Systems, vol. 27, no. 1, pp. 172-180, Feb. 2012. doi: 10.1109/TPWRS.2011.2161349 